\title{
SPACE DISTRIBUTION AND MOTION \\ OF THE LOCAL HI GAS
}

\author{
H. WEAVER \\ Dept. of Astronomy and Radio Astronomy Laboratory, University of California, \\ Berkeley, Calif., U.S.A.
}

In a Joint Discussion devoted to the ages and kinematics of the local stars, inclusion of a paper on the local gas may seem anomalous. There is, however, strong justification for considering such a topic. Newly formed stars retain many properties of the gas from which they originated. To understand the spatial and kinematic properties of local young stars, we must understand the spatial distribution and the kinematics of the local gas from which they were formed.

A cloud within the interstellar gas can collapse gravitationally if its mass, density, and temperature satisfy the Jeans Criterion. Collapse is favored by low temperature and high density.

Various investigators have pointed out that in the Lin Spiral Density Wave Theory a shock must occur on the inner edge of a spiral arm. Such a shock compresses the gas and hence promotes cloud formation with subsequent gravitational collapse to form stars. Shu and several collaborators have shown that such a shock is very effective in triggering cloud formation in a two-phase interstellar medium of the type discussed by Field et al. (1969), and it is widely believed that this is the principal step in the process of forming stars.

Observationally, however, it is found that the majority of local dark interstellar clouds, H I regions, and young stars are located not in the galactic plane, as one would expect from galactic spiral shock theory, but in Gould's Belt, the irregular quasi-planar structure inclined at approximately $20^{\circ}$ to the galactic plane and spoken of earlier in this Joint Discussion by Dr Lindblad. Such observations suggest that in the local region some process other than, or in addition to, the galactic spiral shock process is operative in initiating cloud and star formation. The key to understanding this situation is, I believe, knowledge of how Gould's Belt is related to the local gas out of which clouds and stars form.

In Figure 1, I have employed observations which David Williams and I made to show the column density of local neutral hydrogen over a large portion of the sky extending from longitude $10^{\circ}$ to $250^{\circ}$ within the latitude range $+30^{\circ}$ to $-30^{\circ}$. On the diagram blackness indicates the column density of local neutral hydrogen. The distribution of local gas is decidedly wispy and filamentary in character. It is quite irregular in latitude extent in different longitudes, with a large extension to latitude $-45^{\circ}$ in the longitude range $150^{\circ}$ to $210^{\circ}$. There are many interesting details worthy of comment on this picture, but our purpose here is to examine the overall structure of the local gas. On that scale, the local gas clearly maps onto the sky in the roughly 


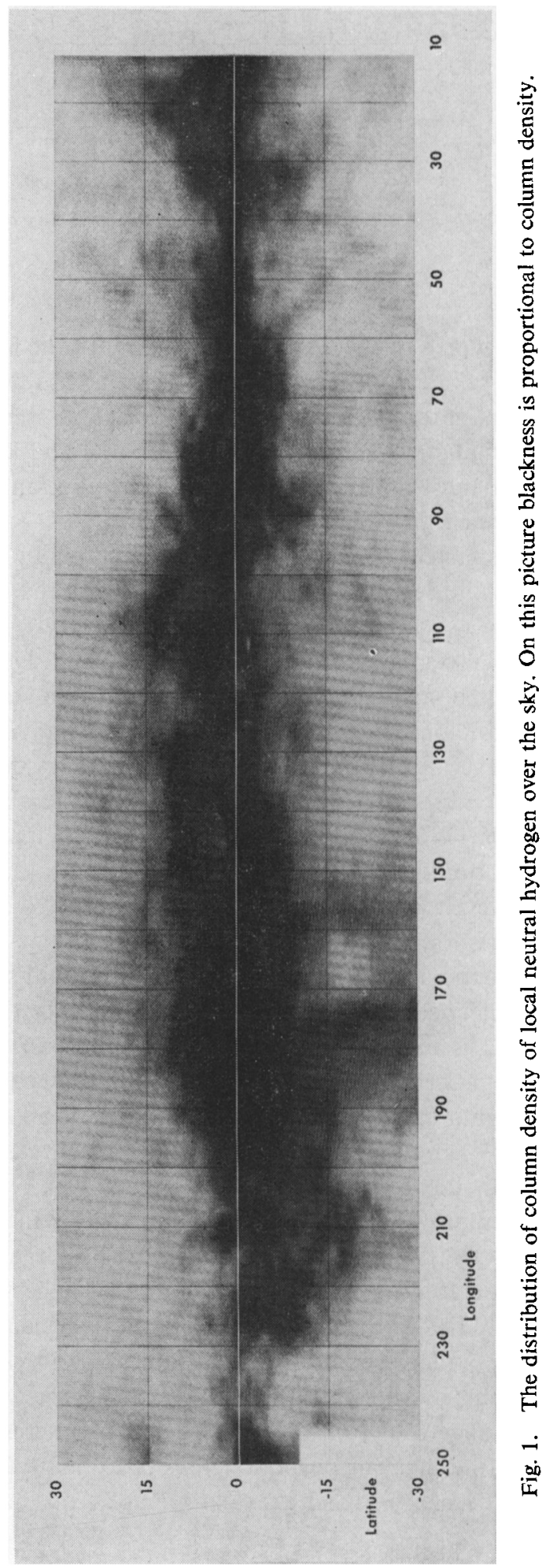


sinusoidal pattern that characterizes Gould's Belt. For example, at $l=200^{\circ}$, the column density at $b=-15^{\circ}$ is five times as great as it is at $b=+15^{\circ}$.

It has long been known that a high degree of correlation exists between the sky distribution we see on this diagram and the sky distribution of dark clouds, $\mathrm{H}_{\mathrm{I}}$ regions, nearby OB supergiants, and young stars in general. All local young objects are closely associated in space within the irregular gas structure we see illustrated in this slide. Since these young objects form from the gas, it is this gas structure that is the basis of Gould's Belt. The distribution of local young stars, for example is strongly associated with the great southward extension of the gas in the longitude range $150^{\circ}$ to $210^{\circ}$.

Lindblad (1967) has shown that in the local neighborhood what he describes as a ring of gas is expanding in the region of the galactic plane. Hughes and Routledge (1972) provided additional evidence for such an expanding ring. Lindblad et al. (1973) have recently improved the numerical parameters originally used by Lindblad to describe the ring. All investigators have associated this expanding ring with Gould's Belt and with the expansion of the local group of early-type stars discussed by Blaauw (1956), by Bonneau (1964), and by Lesh (1968).

Figure $2 a$, taken from the paper by Lindblad and his collaborators, illustrates the nature of the Lindblad hypothesis. The plane of the diagram is the galactic plane; the direction to the galactic center is along the negative $X$-axis; galactic rotation is towards the right. From the origin of the coordinate system a ring of gas extends uniformly at a speed of, say, $4 \mathrm{~km} \mathrm{~s}^{-1}$. After $60 \mathrm{~m}$.y., the ring of gas occupies the position shown, with the sun in the interior of the ring at the position marked by the cross. The ring is elliptical in form because of the combination of expansion and differential galactic rotation operative on the gas.

To an observer at the Sun, the velocity of the ring would, as a function of longitude, appear as shown on Figure $2 b$ as a solid curve. The observational points derived by Lindblad and his collaborators are shown on the diagram. From these points they locate the center of expansion at a distance of $140 \mathrm{pc}$ in the direction $l=150^{\circ}$. The expansion velocity is $3.6 \mathrm{~km} \mathrm{~s}^{-1}$; the expansion age is $60 \mathrm{~m} . \mathrm{y}$. They find a small motion of the center with respect to the local standards of rest.

The extensive Berkeley observations of neutral hydrogen which David Williams and I have made provide the material for investigating the local hydrogen in considerable detail. Figure 3 shows as a function of longitude the velocity distribution of all neutral hydrogen encompassed in the galactic latitude range $-20^{\circ}$ to $-30^{\circ}$. This particular rather high latitude range was chosen for the illustration because (i) it is substantially free from contamination by any non-local gas, and (ii) it crosses a strong section of Gould's Belt at $l=200^{\circ}$.

We immediately note from this diagram that the average velocity of the gas is positive and, in particular, in the vicinity of $l=0^{\circ}$ and $180^{\circ}$ the velocity of the gas is positive. Both these aspects of the velocity distribution denote expansion of the gas.

In Figure 4 mean velocity points derived from this distribution are superimposed on the theoretical curve computed by Lindblad and his collaborators. The agreement 


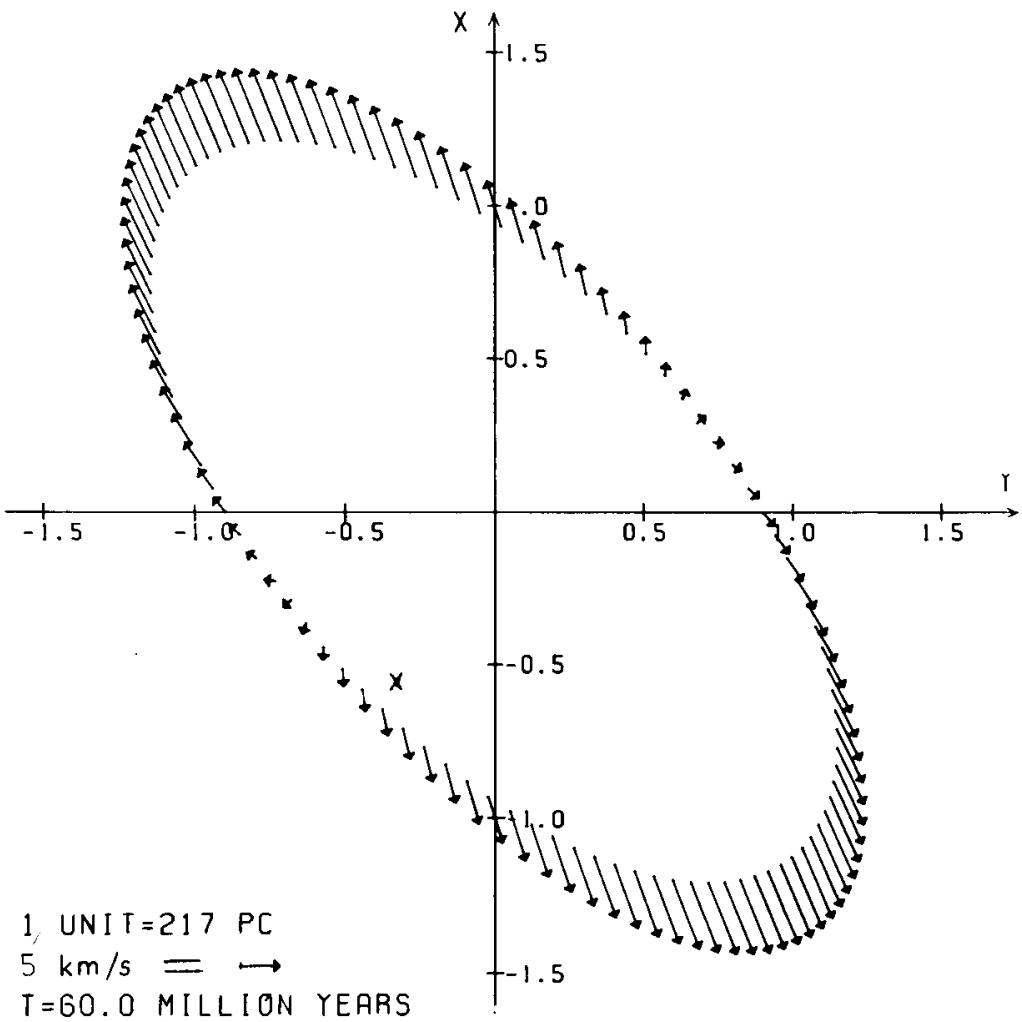

Fig. 2a. The velocity distribution investigated by Lindblad. The gas expanded uniformly from the origin of coordinates. The position of the Sun is marked by the cross. The direction to the galactic center is along the negative $X$-axis. See text for further explanation.

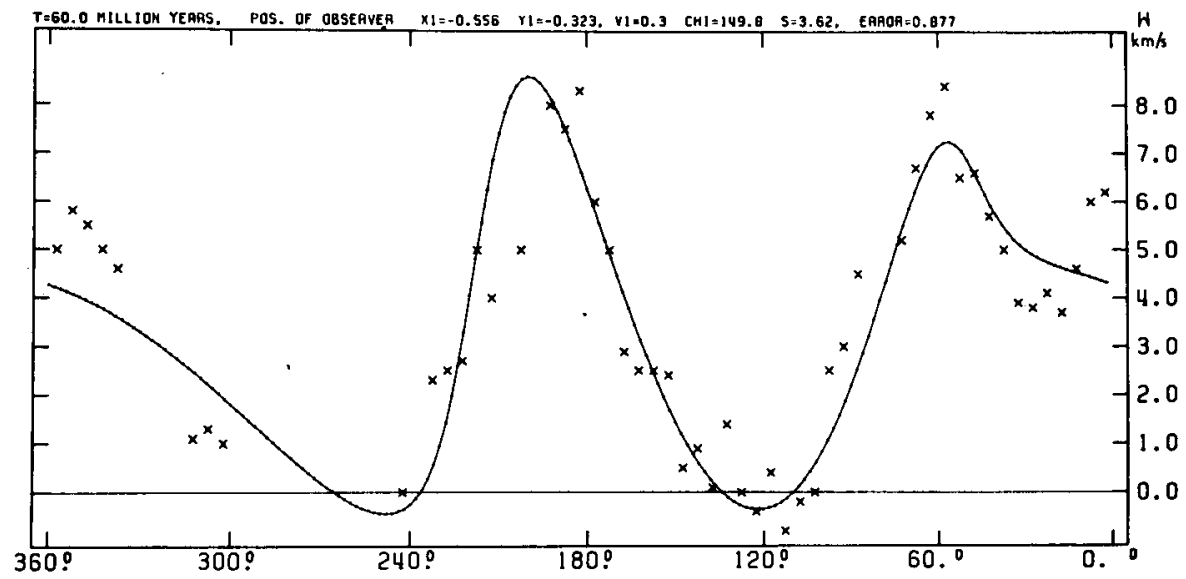

Fig. 2b. The theoretical velocity-longitude diagiam for the velocity distribution shown in Figure 2a is shown by the solid line. Observational values of velocity derived by Lindblad et al. from the neutral hydrogen data are shown as crosses. 


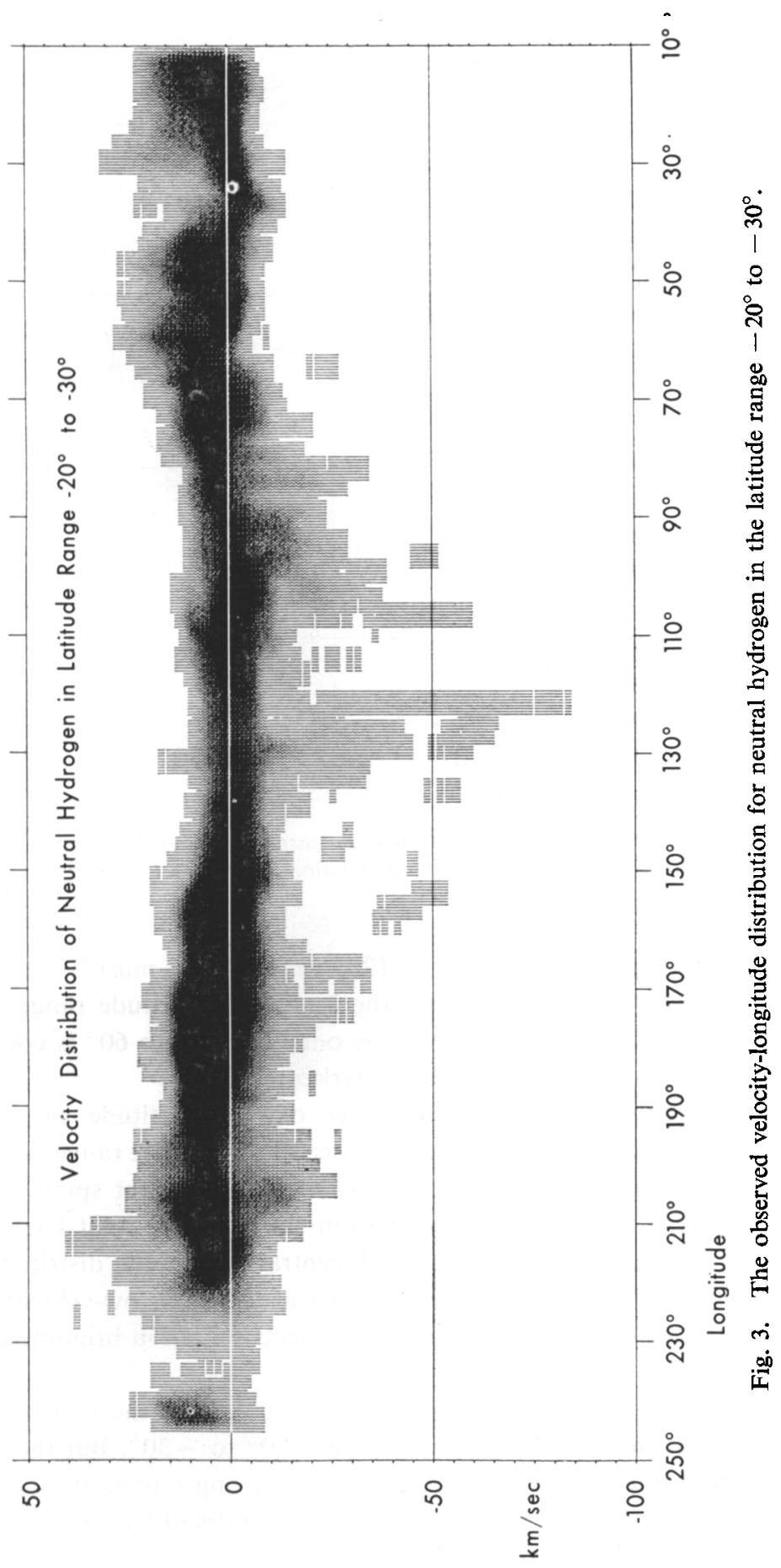




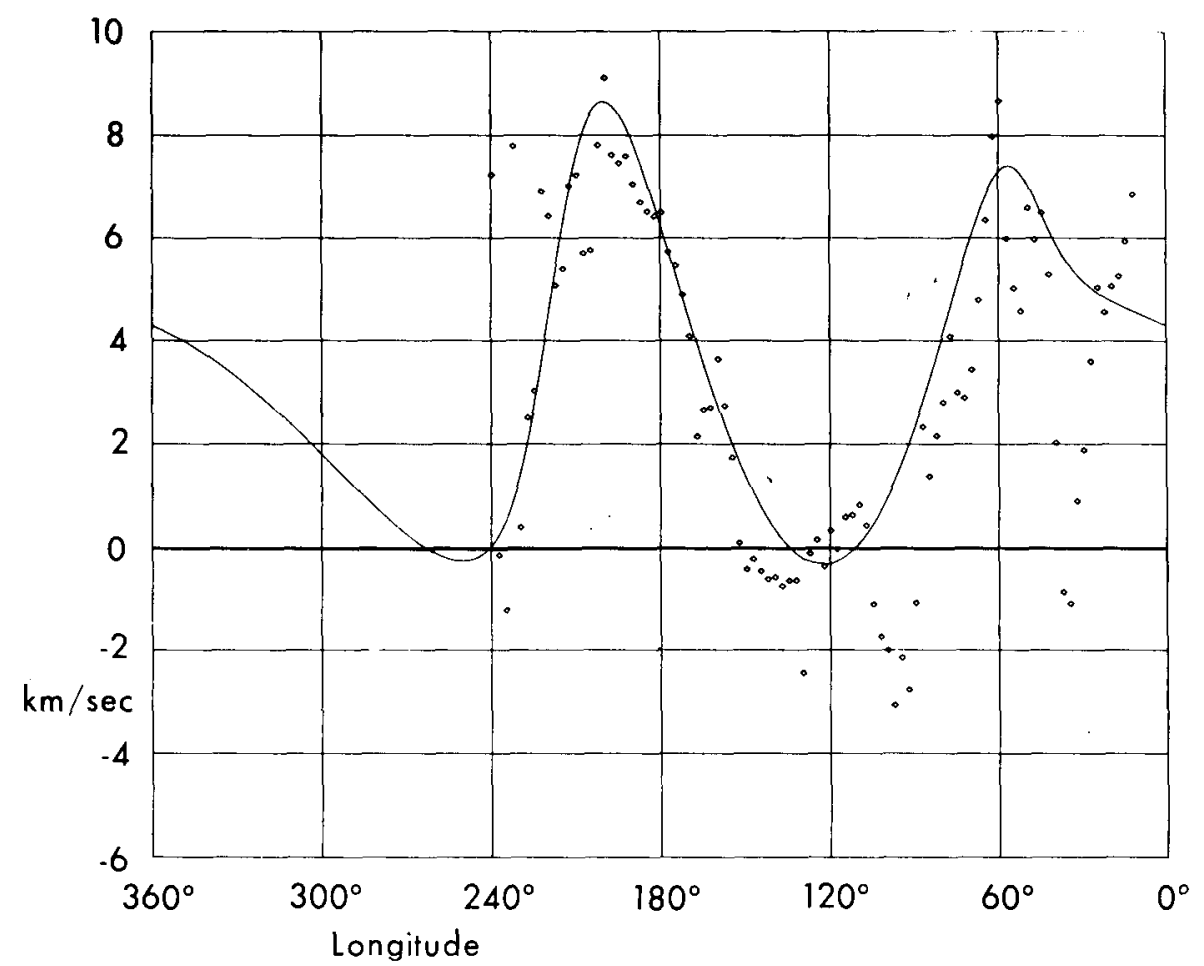

Fig. 4. Mean velocity points for the velocity-longitude distribution shown in Figure 3, superimposed on the theoretical velocity-longitude relation derived by Lindblad et al.

is impressive particularly in the range $l=120^{\circ}$ to $240^{\circ}$, and must be taken as strong confirmation of the basic Lindblad hypothesis. In the longitude range $30^{\circ}$ to $120^{\circ}$ there are local perturbations in the gas. The one from $30^{\circ}$ to $60^{\circ}$ is connected with the north galactic spur and is particularly marked.

In Figure 5 we examine, as representative of lower latitude gas, the velocity distribution of the neutral hydrogen encompassed in the latitude range $+10^{\circ}$ to $+20^{\circ}$. Here there is some contamination from gas lying over distant spiral arms at high $z$-distances. In the diagram this contamination shows as the light background (denoting low intensity) against which the dark central part of the distribution is seen. The local gas (in which we are interested) shows as the dark low-velocity concentration in this figure. Darkness, of course, here respects radiation brightness or column density.

The end regions of this distribution are very similar to the end regions of the distribution we just saw for the latitude range $-20^{\circ}$ to $-30^{\circ}$. But the central part of the distribution illustrated in Figure 5 shows a strong representation of negative velocities in the longitude range $90^{\circ}$ to $200^{\circ}$. The Lindblad hypothesis as originally proposed does not predict such negative velocities.

The suggested explanation of this phenomenon follows from the model shown in 


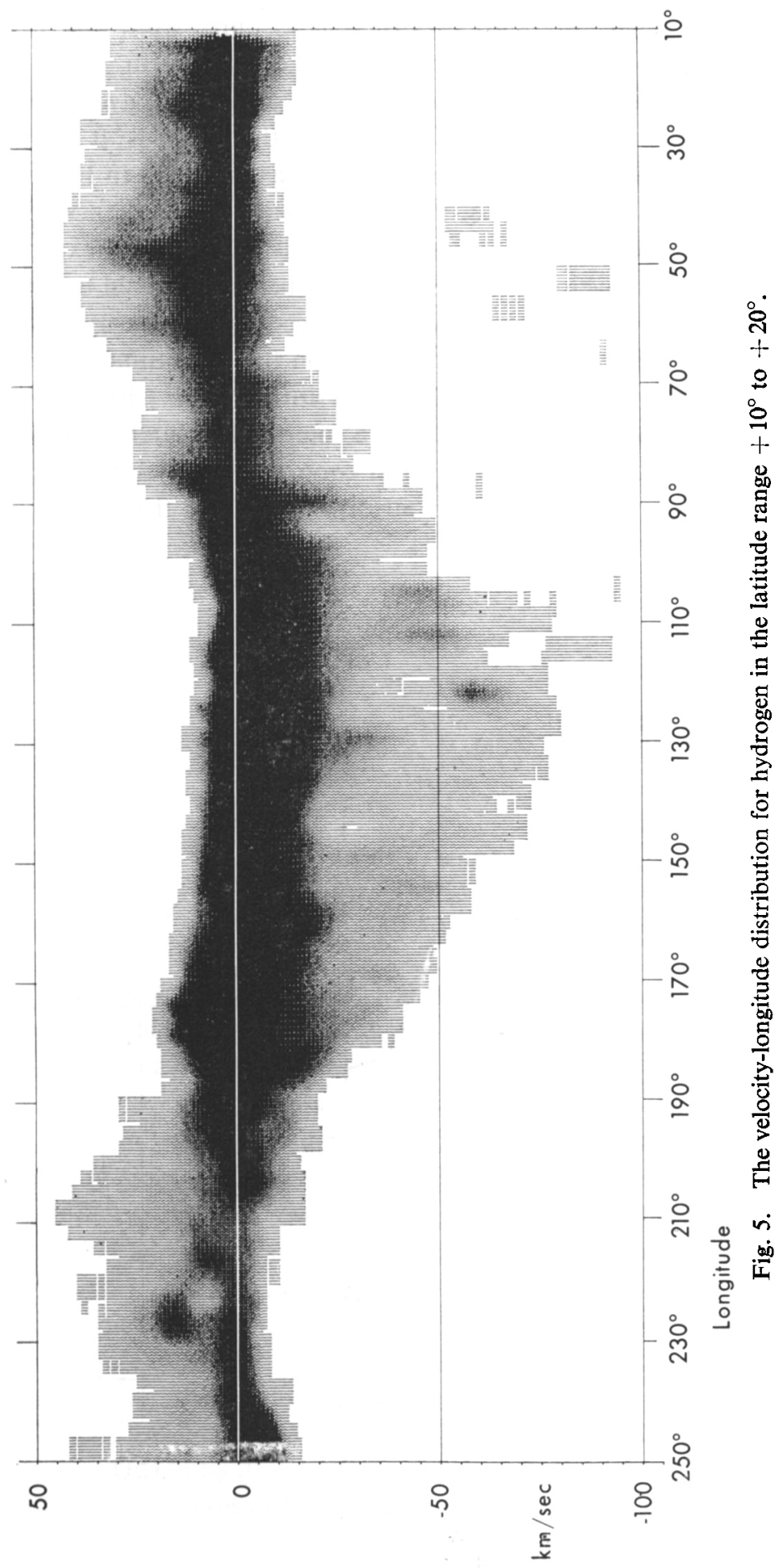


Figure 6a. At the origin of coordinates there is a source from which gas flows uniformly and continuously. After the passage of $12,24, \ldots 60 \mathrm{~m} . \mathrm{y}$. and so on, the gas reaches the boundaries indicated. The density of crosses in any region indicates the relative density of gas at that point. Seen from the sun, gas at each of these loci representing different ages would show velocities as indicated in Figure 6b.

If the flow of gas is continuous, then the velocity distribution we will observe is the envelope of these separate curves. Within this envelope the expected density of points in the longitude-velocity distribution will be proportional to the areal density of crosses on the velocity curves. Taking into account the density of crosses, we see that the predicted velocity-longitude distribution for a continuous flow is strikingly similar to that illustrated for the gas in the latitude range $+10^{\circ}$ to $+20^{\circ}$ shown in Figure 5. The observed distribution shows a somewhat greater extent in the negative velocity direction than the simple uniform continuous model predicts. The real gas flow may not be entirely uniform in all directions. For the present, however, we

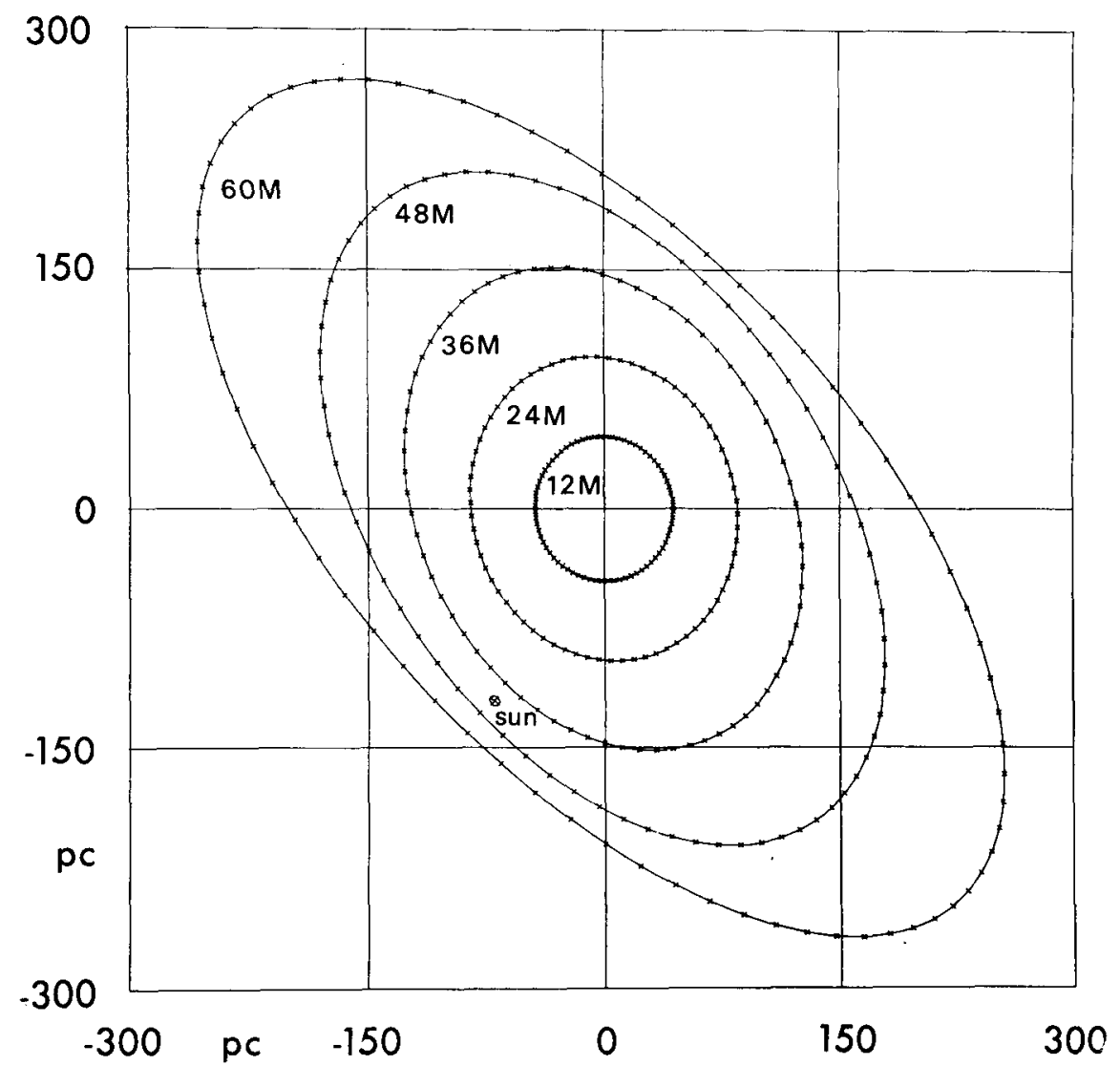

Fig. 6a. Results from a model calculation. Loci occupied by material flowing from the origin of coordinates after periods of $12 \times 10^{6}, 24 \times 10^{6}, \ldots, 60 \times 10^{6} \mathrm{yr}$. Differential galactic rotation is present as well as uniform outflow from the origin. The position of the Sun is indicated. The direction towards the galactic center is as in Figure 2a. See text for explanation of the crosses on the various loci. 


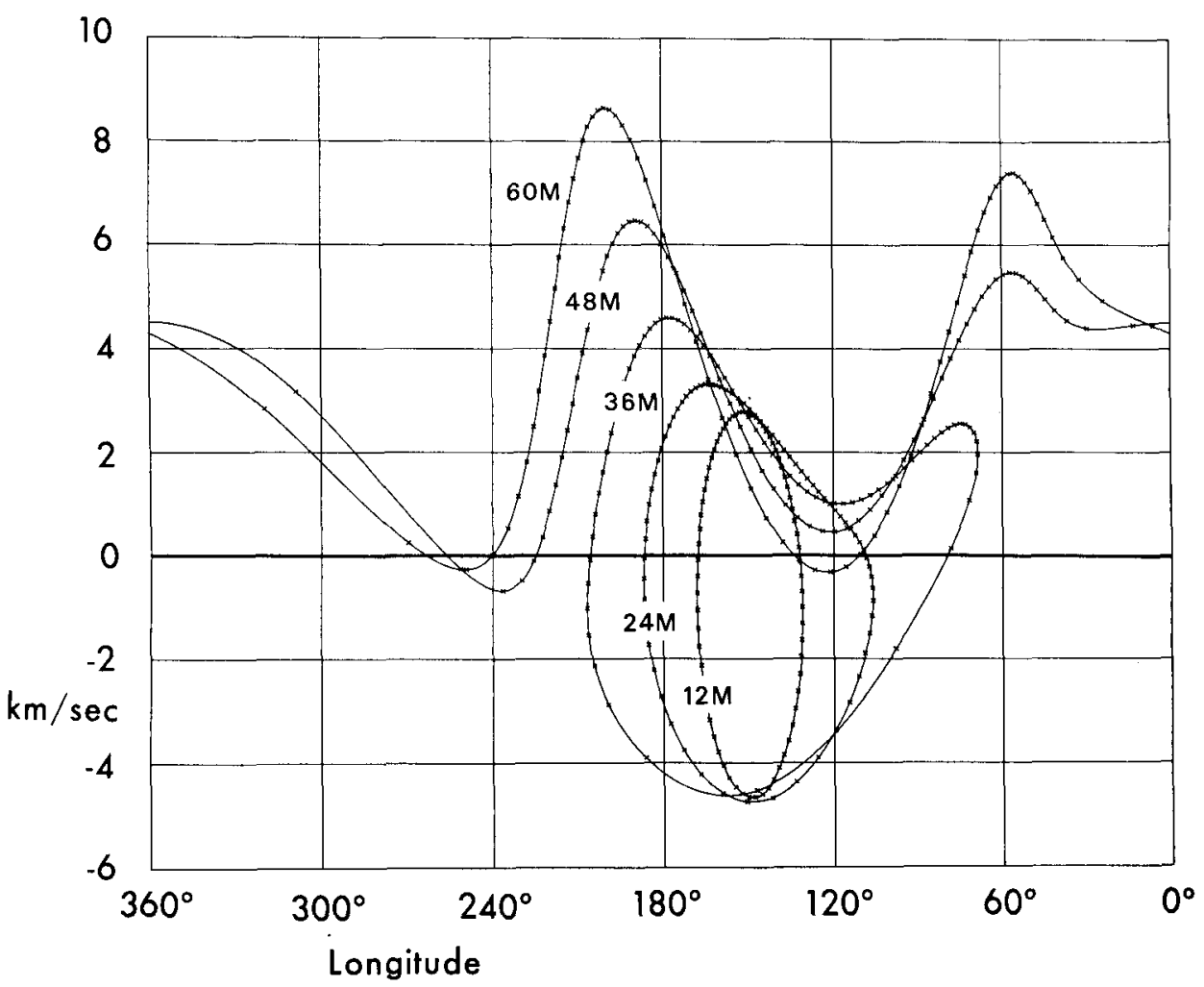

Fig. 6b. Velocity-longitude diagrams for the loci shown in Figure 6a.

ignore this slight irregularity in discussing a theoretical model. We adopt as our model of the gas flow in Gould's Belt a uniform continuous flow from a source located at a distance of approximately $140 \mathrm{pc}$ in longitude $150^{\circ}$. When we look in the latitude range $-120^{\circ}$ to $-30^{\circ}$, we look above much of the continuous flow; we see the periphery of the flow, which might be described as an expanding ring. At lower latitudes our line of sight encounters the continuous flow pictured in the model.

If such a continuous flow of gas exists in Gould's Belt, and generally near the galactic plane, other objects clearly associated with the gas in the Belt must show that flow also.

The local dark clouds have long been known to be closely associated with Gould's Belt. We should thus expect the velocity distribution of these clouds to lie within the envelope of the velocity distribution representative of the local gas. The velocities of the clouds need not, of course, uniformly fill that envelope. There is no reason to expect that the clonds will be distributed uniformly throughout the gas.

Dieter (1973) has observed velocities for more than 100 dark clouds from their formaldehyde absorption lines at $4830 \mathrm{MHz}$. The velocity distribution of the dark clouds is shown in Figure 7 as a function of longitude. The dark cloud velocities are 


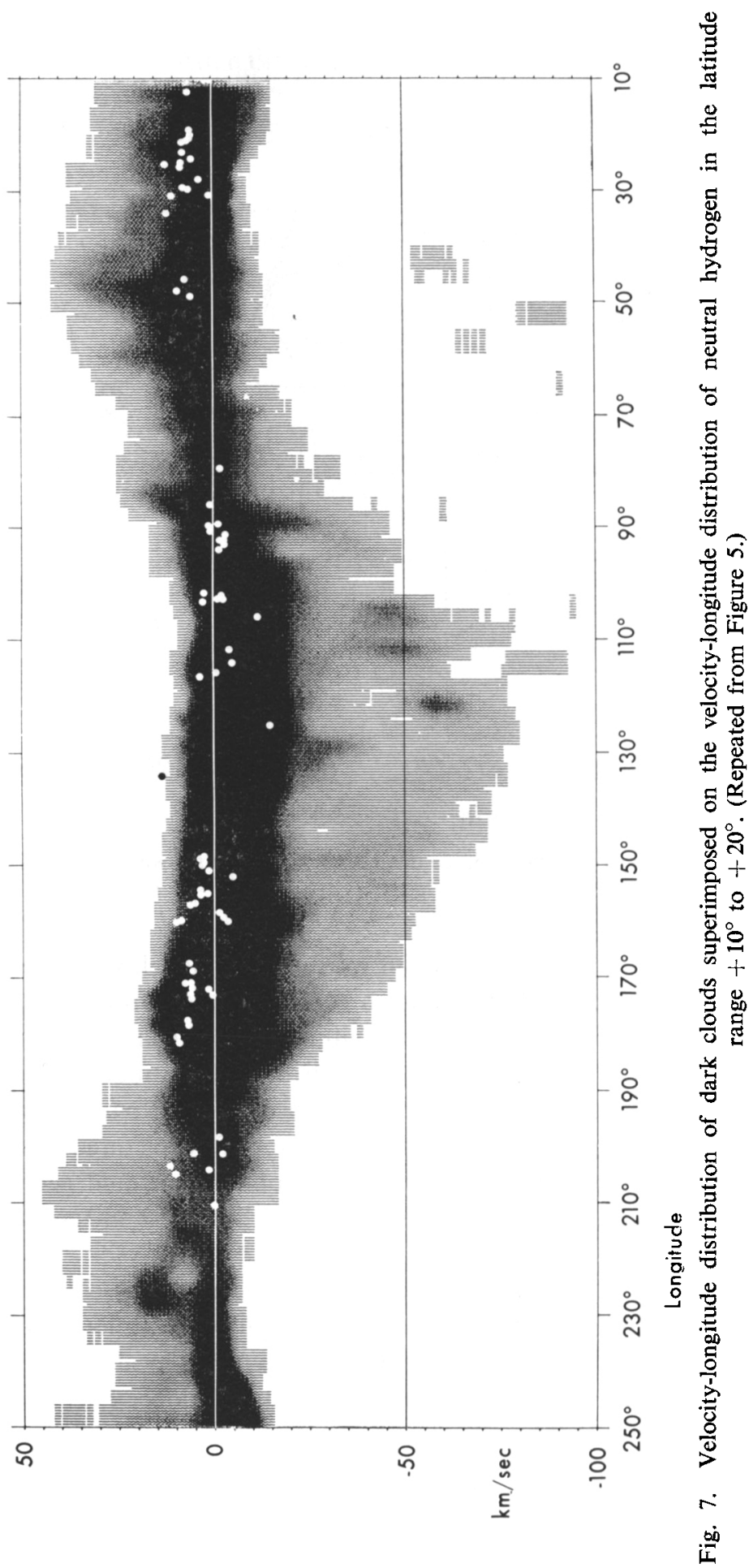




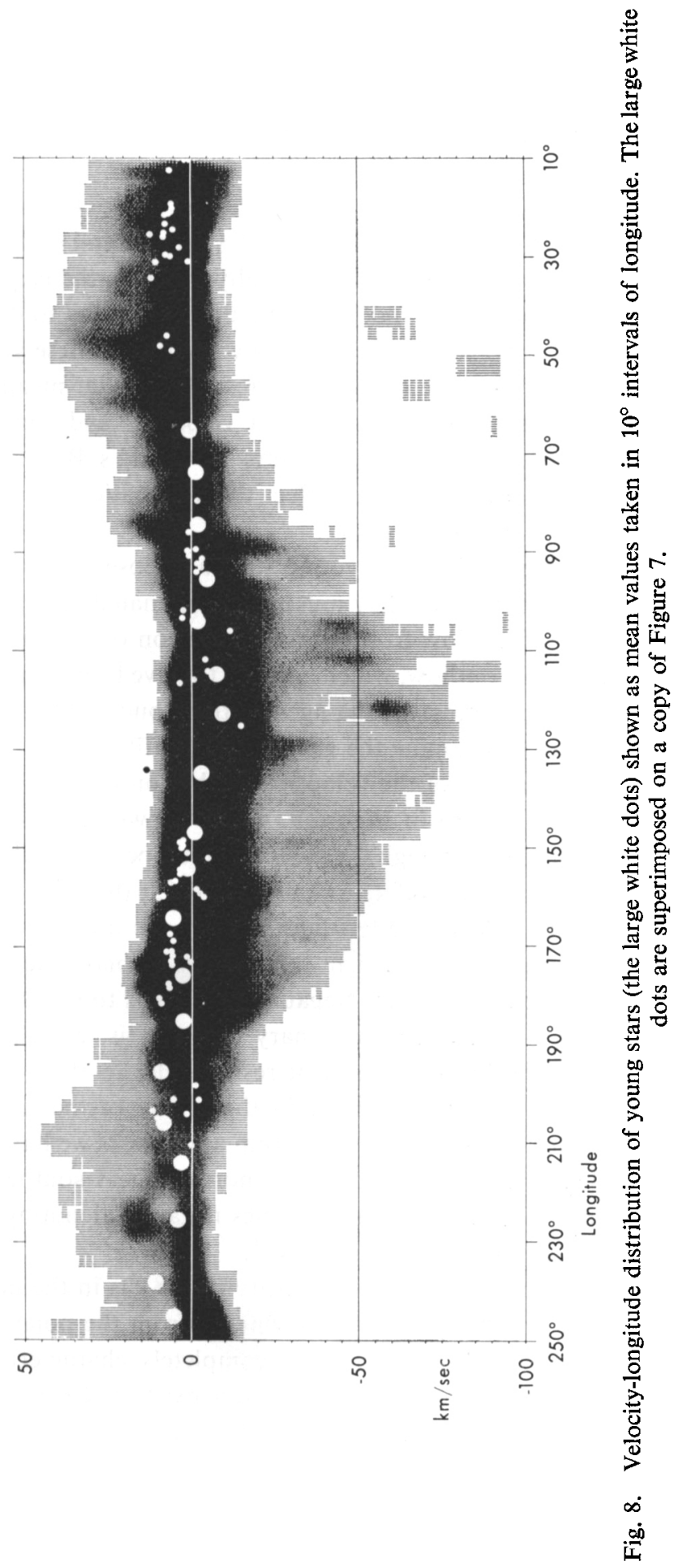


shown as dots against the background of the velocity distribution of the local gas. The dark clouds show a velosity distribution characteristic of an expanding group. There are, for example, positive velocities in the regions $l=0^{\circ}$ and $180^{\circ}$. The velocity distribution of the dark clouds lies within the envelope of the velocity distribution of the local gas as we might have anticipated.

Young stars are closely associated with the gas and dark clouds in Gould's Belt. Lesh (1968) has discussed the kinematics of young stars, B5 and earlier, within $600 \mathrm{pc}$ from the Sun. The population she studied spatially overlaps the gas in which we are interested between longitudes $60^{\circ}$ and $250^{\circ}$. We limit distances to $400 \mathrm{pc}$. In Figure 8 we see represented as very large dots the mean velocities of these young stars taken in $10^{\circ}$ intervals over the longitude range of interest. The stellar mean velocities - the large dots - lie closely among the dark cloud velocities and well within the gas velocity distribution envelope. The young stars in Gould's Belt also form an expanding group like the dark clouds and reflect the kinematic properties of the local gas.

The local dark clouds and the local young stars are closely associated in Gould's Belt with the local gas from which they must have originated. They all show a common kinematic pattern of outflow from a specified region of the galactic plane.

What is the source of this outflow, which appears to have been surprisingly constant over a time scale at least as great as the ages of the clouds and stars we have considered? For an answer, we examine the gas at higher latitudes.

An example of the character of the velocity distribution of local neutral hydrogen at high galactic latitudes is seen in Figure 9, which shows the velocity distribution for all the gas in a great circle through the galactic poles, perpendicular to the galactic plane. The left side of each figure refers to longitude $49^{\circ} .5$; the right hand side of each figure refers to longitude 229.5 . The left figure shows the directly observed velocity distribution of the hydrogen gas; the right-hand figure displays the mean velocity of the hydrogen as a function of latitude. Negative velocity is towards the center of the circle, positive velocity is outwards. Stationary gas, that is, gas showing no radial motion with respect to the local standard of rest, would lie along the zero-velocity circle. Note that the gas is not stationary. In the region of the galactic plane, the average velocity is positive, denoting outflow from a source as we have already found. As we go towards higher latitudes in both the northern and southern hemispheres, the mean velocity goes through zero and becomes negative, denoting downflow. The mean downflow is at least as great as $10 \mathrm{~km} \mathrm{~s}^{-1}$.

The phenomenon of universal negative velocity of the gas in the polar regions has long been known. The velocity pattern of downflow from the polar regions in both hemispheres, and outflow at low latitudes, is completely characteristic of the local region of the Galaxy. A major gas circulation pattern exists in the solar neighborhood.

A model that accounts for all of the observed phenomenons is shown in Figure 10.

There is flow from above the galactic plane in both hemispheres. The two approximately equal streams collide. Gas flows outward, away from the collision region. The fact that the colliding streams are not precisely equal or precisely centered 


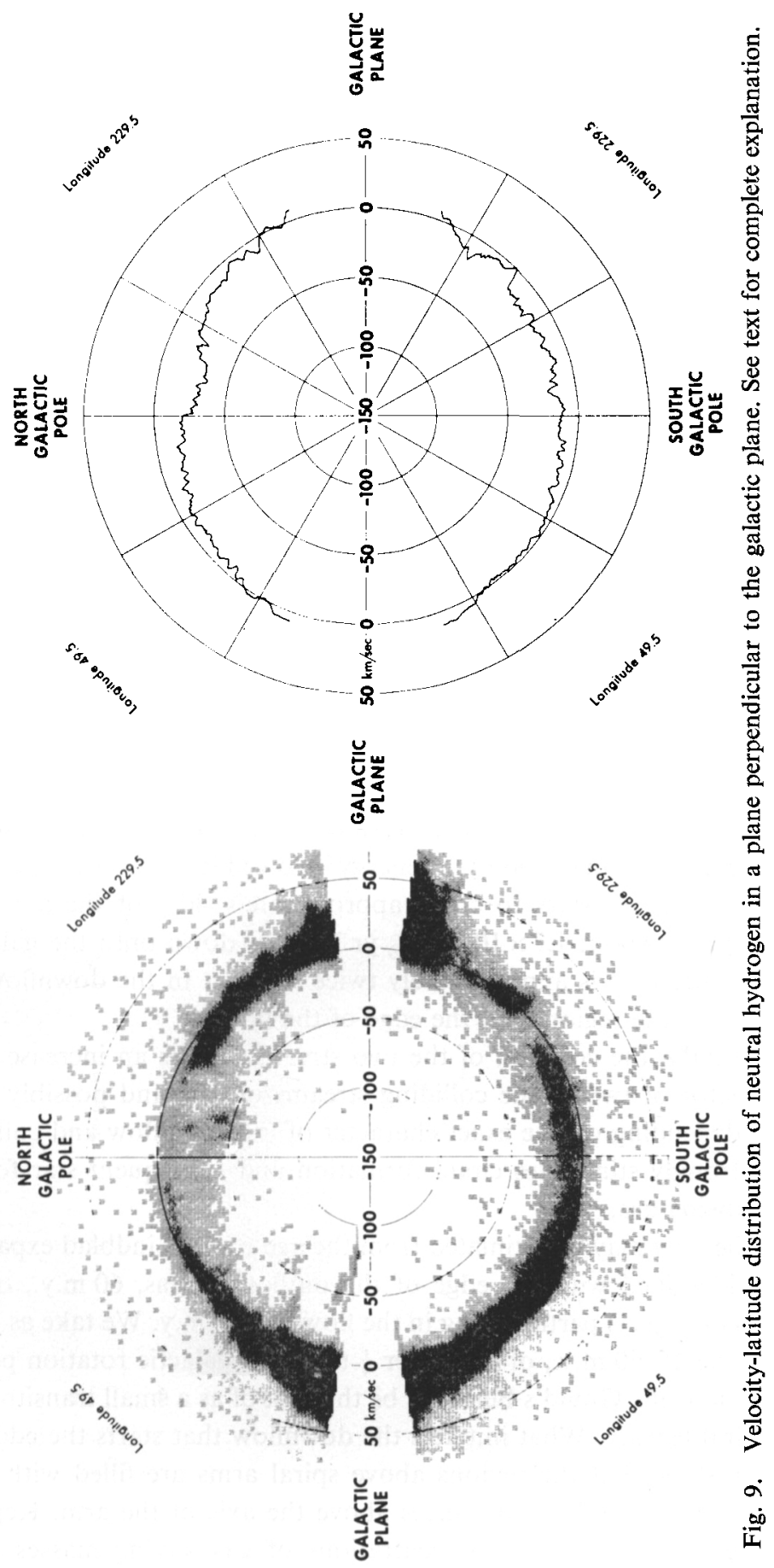




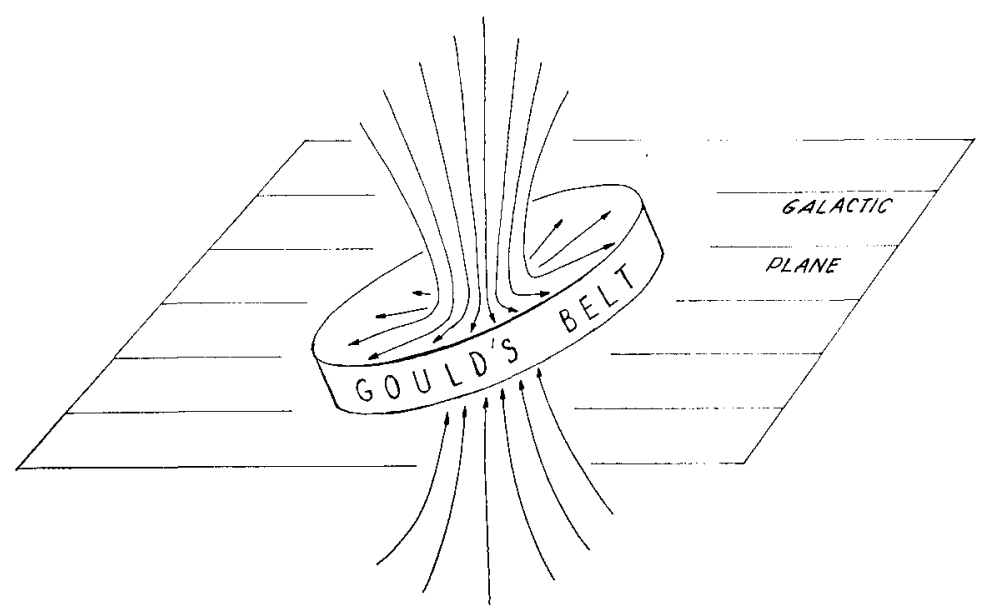

Fig. 10. Model showing schematically the explanation of Gould's Belt.

accounts for the roughly $20^{\circ}$ tilt of the principal plane of the flow which is, of course, Gould's Belt. Irregularities of outflow exist in both velocity and direction as, for example, the great southward extension of the local gas in the longitude range $150^{\circ}$ to $210^{\circ}$. The average half-thickness of the flow is $\sim 200 \mathrm{pc}$, slightly more than one scale height, but the thickness is quite variable from one longitude to another. The outflow is $\sim 4 \mathrm{~km} \mathrm{~s}^{-1}$ but shows variability with direction and location. The downflow velocity is at least $10 \mathrm{~km} \mathrm{~s}^{-1}$, and probably rather higher. About $10^{6}$ solar masses of hydrogen are involved in the flow; the energy involved is of the order $10^{50}-10^{51}$ ergs. The downflowing gas appears to cover approximately $40 \%$ of the area of the expanding gas region. About $1.7 \times 10^{-2} M_{\odot} \mathrm{yr}^{-1}$ flows down onto the galactic plane. The gas density per $\mathrm{cm}^{3}$ is approximately twice as great in the downflow region of the plane as it is in the region near the edge of the flow.

Most importantly, the collision of the two streams causes an increase in pressure of at least a factor of two in the colliding streams of gas, and possibly by a much greater factor depending on the exact character of the downflow and collision. Such overpressure strongly stimulates cloud formation and subsequent star formation in the outward flowing gas.

The age of the flow may be estimated from the age of the Lindblad expanding ring, which is here identified with the edge of the outflow of gas, $60 \mathrm{~m} . y$., or from the expansion ages of objects participating in the flow, 45-90 m.y. We take as an estimate of the age a value $45-60 \mathrm{~m}$.y., of the order $\frac{1}{4}$ to $\frac{1}{3}$ of a galactic rotation period.

On the galactic scale, Gould's Belt may be thought of as a small transitory gas eddy in the local spiral feature. What initiates the downflow that starts the eddy?

Observations show that the regions above spiral arms are filled with substantial masses of gas at considerable $z$-distances above the axis of the arm. Kepner (1970) and other observers have found concentrations of gas having masses as great as $0.5-1 \times 10^{6} M_{\odot}$ at $z$ distances greater than $1 \mathrm{kpc}$. 


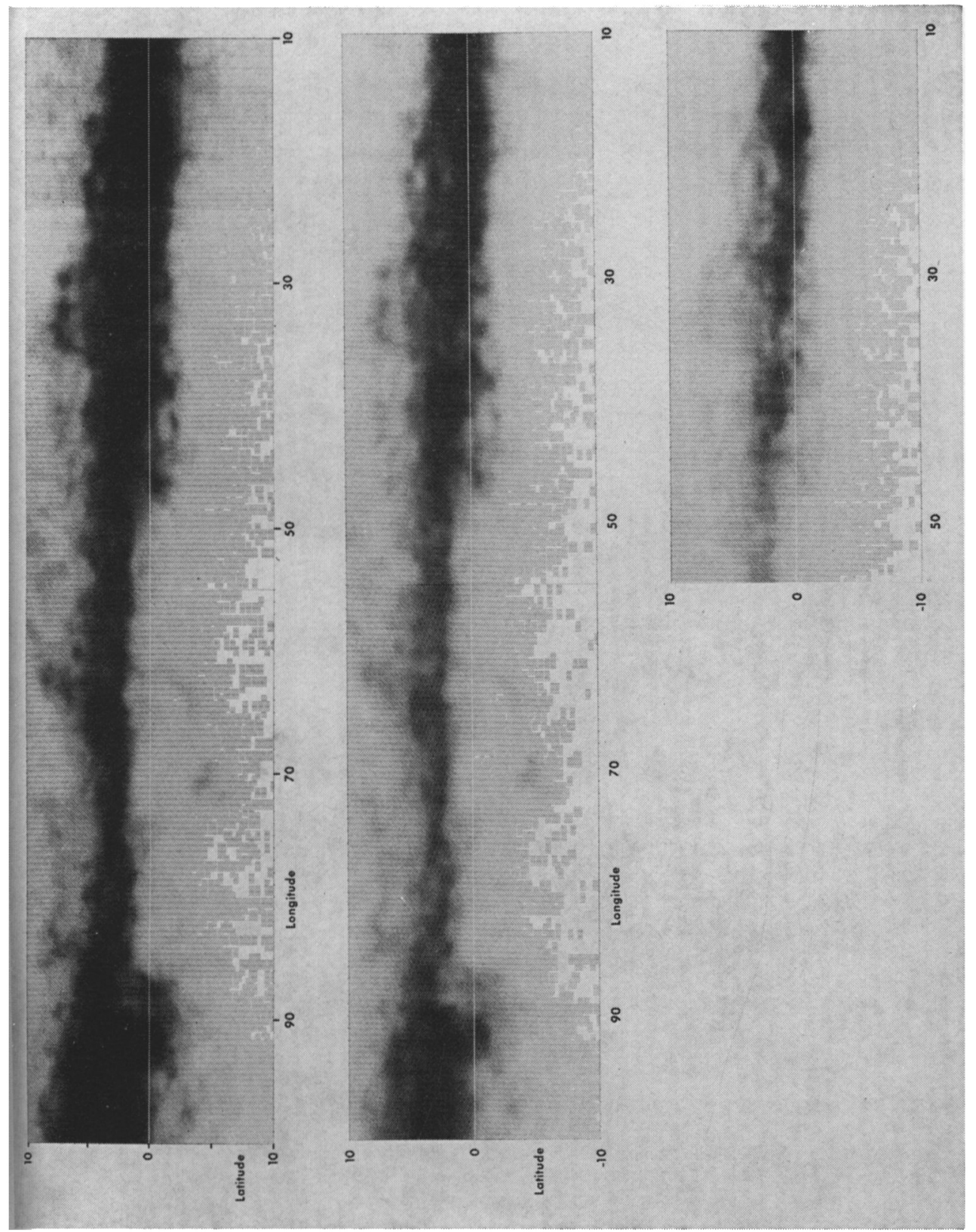

붕 品 娄 的焉 需 $\dot{5}:$ 鄢 范苛寻 总星 空 蔗 हี

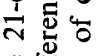
导要 $\Xi$ 宁 实造 홍 \& ธี ริ옹 㐘 os 00 들. \% 드 해웡

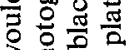
站主. 늘 क जु $>0$ 品 ब응 贾

号害

$\mp$ 品 넝 음 ฐ ๘ 항 崖 ठ음 F $\Xi \dot{\Xi}$

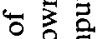
的 $\Xi 0$ 농 ㅍ. 8 品 专 曹 현흥 这 品 흥 ช 4 豆。 .

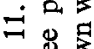
的点穿 
Figure 11 shows a computer-produced picture of the outer arm of the Galaxy as it appears on the sky in neutral hydrogen radiation. The large amount of gas present in the form of overlying cloud-like structures is evident. Many features are a kpc above the axis of the arm; a great many are at heights of 400 to $500 \mathrm{pc}$. Masses of many of these features are $\sim 10^{6} M_{\odot}$. Such features above the arms cannot be permanent. They must be buoyed up from the arm for a short time and then return to the plane, guided, perhaps, by the buoyed-up or inflated magnetic lines as in a Parker-type instability. The velocity of downfall we observe in the solar neighborhood corresponds to free-fall from $z$-heights of the order of $300-400 \mathrm{pc}$.

One may suggest, then, the following schematic picture of the gas in the solar neighborhood. Masses of gas at moderate $z$-distances above the plane become unstable and fall back to the plane. Two streams of descending gas, one from above the plane, the other from below, flow onto the plane, collide, and form the Gould's Belt gas structure we observe. The tilt of the gas structure and irregularities within it are caused by density and velocity irregularities in the colliding streams of gas. The collision of the down flowing gas streams compresses the gas and promotes the formation of clouds with subsequent gravitational collapse to form stars. The stars formed from the gas reflect the spatial and kinematic properties of the gas. The gas flow has existed for only a small fraction - perhaps $\frac{1}{4}$ to $\frac{1}{3}-$ of a galactic rotation period. We may expect that in a further small fraction of a rotation period, this small local eddy in the gas will damp out and disappear, leaving behind, for a much longer time, the group of aging stars to which it gave birth.

\section{References}

Blaauw, A.: 1956, Astrophys. J. 123, 408.

Bonneau, M.: 1964, J. Obs. 47, 251.

Dieter, N. H.: 1973, Astrophys. J. 183, 449.

Field, G. B., Goldsmith, D. W., and Habing, H. J.: 1969, Astrophys. J. Letters 155, L149. (See also

Goldsmith, D. W., Habing, H. J., and Field, G. B.: 1969, Astrophys. J. 158, 173.)

Hughes, V. A. and Routledge, D.: 1972, Astron. J. 77, 210.

Kepner, M.: 1970, Astron. Astrophys. 5, 444.

Lesh, J. R.: 1968, Astrophys. J. Suppl. Ser. 17, 371.

Lindblad, P. O.: 1967, Bull Astron. Inst. Neth. 19, 34.

Lindblad, P. O., Grape, K., Sandqvist, Aa., and Schrober, J.: 1973, Astron. Astrophys. 24, 309.

\section{DISCUSSION}

Van Woerden: Dr Weaver's paper is highly interesting and thought-provoking, but I wish to make two remarks.

(1) There is, in the low-velocity hydrogen, another striking feature in addition to Gould's Belt. Fejes and Wesselius (Astron. Astrophys. 24, 1, 1973) have pointed out two ridges in opposite locations, together outlining a tilted disk ('Scheve Schijf') inclined by $45^{\circ}$ to the galactic plane and with its pole at $l \sim 120^{\circ}, b=45^{\circ}$. This disk may be genetically related to the intermediate-negative-velocity gas discussed by Wesselius and Fejes (Astron. Astrophys. 24, 15, 1973) and to the high velocity clouds.

(2) Weaver's picture of two gas streams flowing in from high latitudes, $|b| \sim 60^{\circ}$ to $70^{\circ}$, towards the centre of Gould's Belt appears overly schematic. At high negative latitudes his stream may well be present. At high positive latitudes, however, the scene is dominated (cf. Blaauw and Tolbert, 
Bull. Astron. Inst. Neth. 18, 405, 1966; Wesselius and Fejes, Astron. Astrophys. 24, 15, 1973) by a big hole in the low-velocity hydrogen and, closely over lapping, a big complex of hydrogen with average velocity $-42 \mathrm{~km} \mathrm{~s}^{-1}$. Wesselius and Fejes show in detail that the hole and the complex are probably related, and that the complex is likely to come, at a speed of $70 \mathrm{~km} \mathrm{~s}^{-1}$, from the direction $l=120^{\circ}, b=+40^{\circ}$. They estimate the distance of this complex at $70 \mathrm{pc}$, its mass at $1800 M_{\odot}$, its energy at $0.9 \times 10^{50} \mathrm{erg}$. The origin of this complex is probably the same as that of the high-velocity clouds, which come from the same direction.

Weaver: Dr van Woerden has introduced the topic that would have been the second part of my talk at this Joint Discussion if I had been allocated another ten or fifteen minutes. I will comment on his second remark first.

Before the appearance of the paper by Wesselius and Fejes, completely independent analysis of the Berkeley Neutral Hydrogen Survey data made without any knowledge of their work, led to precisely the same picture of the hole and the intermediate-negative-velocity gas derived by Wesselius and Fejes. My estimates of distance, mass, and so forth are in excellent - indeed, in several instances, exact - agreement with theirs.

What Dr van Woerden terms a 'complex of hydrogen' I would describe rather more specifically and simply as a mass of hydrogen consisting of $\sim 2 \times 10^{3} M_{\odot}$ moving in a non-circular galactic orbit with small $z$-motion. This mass of gas is now approximately 70 pc north of the galactic plane, and is colliding with the local gas. It probably originated in the next outer arm of the Galaxy, perhaps as much as $2 \times 10^{7} \mathrm{yr}$ ago, when its orbit was changed from circular or near circular to quite elliptical by some energetic event such as a supernova explosion. While internal motions have dispersed the mass over several hundred parsecs during this time interval, it appears to have a fairly compact core that is the most evident colliding mass.

Since the orbit of this gas is non-circular, the gas has a radial component of motion inward towards the galactic center, and it has a lower speed in the direction of galactic rotation than the local gas. We are running into the mass of gas. From our point of view, however, and with respect to the local standard of rest, the mass of gas is like a jet stream moving just over our heads at a distance of $70 \mathrm{pc}$ with a velocity of possibly $70 \mathrm{~km} \mathrm{~s}^{-1}$. It has seriously perturbed a portion of the down-flowing gas; it has punched a hole in a portion of that gas. But the duration of the collision is short, a few percent of the duration of the down flow, and the mass of the colliding gas is very small compared to the mass in the down-flowing stream. The collision now in progress will certainly affect the future development of the evolving structure we call Gould's Belt, but it does not completely obscure the stream of down-flowing gas in the northern hemisphere as Dr van Woerden's remark implies. The downflowing stream is easily visible.

I regret that there is not more time to discuss this interesting topic on which a great deal of information is now available.

In regard to Dr van Woerden's first comment I would remark only that the intriguing 'Scheve Schijf' found by Fejes and Wesselius may well be related to the collision phenomenon I have just been discussing. Clearly, however, the Scheve Schijf is a minor feature on the scale of Gould's Belt; its total mass is a few percent of that of Gould's Belt.

Tolbert: Why should the streams of gas from either side of the galaxy come inward at the same time? The gas distributions away f $f_{1}$ om the plane are very different on either side, and it's not clear to me why these two streams should arise at the same time and in the same place near the Sun.

Weaver: There is no immediate and saisfying answer to Dr. Tolbert's question. I have discussed what is observed and I have tried to synthesize what is observed in the form of an understandable and physically consistent model.

There is at present no clear understanding of the physics of high- $z$ clouds of the sort we saw so clearly over the spiral arm shown on the last slide. We do not understand how such masses of gas rise above the plane, or how their flow back to the plane is initiated.

In view of my discussion of Dr van Woerden's remarks, it should be clear that what may appear as a very different distribution of gas on the two sides of the plane now, may be no more than a momentary perturbation on the time scale of Gould's Belt, which is the time scale of interest in these considerations. What we see now was not the case over the time period of significance in the formation of Gould's Belt.

Murray: It seems remarkable that the suspected high concentration of young $M$ stars in the north galactic cap is just where your gas density is least; whereas in the south, the gas density is more dense and there may not be as many low velocity $M$ dwarfs. 
Weaver: The gas density in the north galactic cap is normal over the $z$-range 0 to approximately $70 \mathrm{pc}$. The young $\mathrm{M}$ dwarfs to which Dr Murray refers are, I believe, closer to the Sun than $70 \mathrm{pc}$ on at least no farther away than $70 \mathrm{pc}$. I see no suggestion of a discrepancy that the stars are found in a region of low gas density as implied by Dr Murray's question.

We should also keep in mind that 'young' is a relative term. Gould's Belt is young, perhaps $60 \times$ $10^{6} \mathrm{yr}$ old, the collision (and the resultant low gas density above the plane) discussed earlier is young, perhaps $2 \times 10^{6}$ yr old. How old are the $M$ dwarfs?

Clube: Would you like to comment on the fact that A stars are moving towards us with the same velocity as the HI? It seems to me that the idea of gas streams contained by magnetic fields may be difficult to uphold.

Weaver: It is not my recollection that the A stars are moving with such velocity. The down-flow velocity of the gas is 10 or more $\mathrm{km} \mathrm{s}^{-1}$. The radial velocities of $A$ stars in the north galactic polar cap were observed by Perry (Astron. J. 74, 139, 1969). His results indicate for A stars within approximately $25^{\circ}$ of the NGP a mean radial velocity of essentially zero. He did find, in the course of his investigation, that the A stars appear to form two populations which have different kinematics properties. The physical meaning of this fact remains to be clarified, but it does not appear to indicate a discrepancy of the sort mentioned by Dr Clube.

We do not understand the physics of the high- $z$ concentrations of gas observed to lie above the spiral arms - how the gas gets there or what triggers the process by which it falls back to the plane. A suggestion made some time ago by Parker in a different context is that gas in the spiral arms may be buoyed up by the pressure of cosmic rays and may slide back to the plane along magnetic lines of force in the arms. I do not propose this as the mechanism operative here; I suggest that it is one possibility that is well established in the literature and that we should consider in the present context. 